\title{
INSTRUMENTOS DE AVALIAÇÃO COM ENFOQUE EDUCACIONAL: REVISÃO SISTEMÁTICA
}

ASSESSMENT TOOLS WITH EDUCATIONAL FOCUS: SYSTEMATIC REVIEW
INSTRUMENTOS DE AVALIACIÓN CON ENFOQUE EDUCACIONAL: REVISIÓN SISTEMÁTICA

\author{
Michele Oliveira da Silva* \\ Eduardo José Manzini**
}

\begin{abstract}
Resumo: O uso de instrumentos pode auxiliar o professor na avaliação de diversas áreas no âmbito educacional. No entanto, muitos dos instrumentos publicados restringem seu uso à formação do profissional da área da saúde. Diante disso, o objetivo desta pesquisa foi identificar instrumentos de avaliação com enfoque educacional para uso de profissionais formados em Pedagogia. Optou-se por realizar uma revisão sistemática em duas bases de dados (uma de origem estrangeira e outra de origem brasileira). Inicialmente, foi realizada uma seleção dos artigos a partir de critérios de inclusão e exclusão da pesquisa. Em seguida, a partir da leitura desses artigos selecionados, foram identificados instrumentos de avaliação utilizados na metodologia das pesquisas. Como resultado, foram encontradas 21 referências de instrumentos de avaliação com origem estrangeira e nove referências de instrumentos de avaliação com origem nacional, a partir dos quais um breve perfil foi traçado. A metodologia utilizada nesta pesquisa contribuiu para identificar os instrumentos de avaliação existentes e disponíveis para uso do pedagogo e para evidenciar como a revisão sistemática pode ser utilizada nesse tipo de pesquisa.
\end{abstract}

Palavras-chave: Educação. Instrumentos de avaliação. Revisão sistemática.

Abstract: The use of tools can help teachers in assessing several areas in the educational field. However, many of the published tools restrict its use to the training of health care professionals. Thus, the objective of this research was to identify assessment tools with educational focus to be used by Pedagogy professionals. A systematic review of two major databases was conducted to identify assessment tools from national and international publications. Firstly, the articles were selected to decide by inclusion and exclusion criteria of the study. After that, these articles were analyzed and from their reading, the assessment tools used in research methodology were identified. As a result, twenty one international references regarding assessment tools and nine Brazilian references were found. A brief profile of the tools was drawn. The methodology used in this research has contributed in identifying the existing assessment tools available for the pedagogy professional, and in emphasizing how this systematic review has contributed to this type of research.

Keywords: Education. Assessment tools. Systematic review.

Resumen: El uso de instrumentos puede auxiliar el profesor en la evaluación de diversas áreas en el ámbito educacional. Entretanto, muchos de los instrumentos publicados restringen su uso a la formación del profesional del área de la salud. Delante de eso, el objetivo de esta pesquisa fue identificar

\footnotetext{
* Universidade Estadual Paulista - UNESP - Campus de Marília. E-mail: michelepedagogia@yahoo.com.br

${ }^{* *}$ Universidade Estadual Paulista - UNESP - Campus de Marília. E-mail: manzini@marilia.unesp.br
} 
instrumentos de evaluación con enfoque educacional para uso de profesionales formados en pedagogía. Se optó realizar una revisión sistemática en dos bases de datos (una de origen extranjera y otra de origen brasileña). Inicialmente fue realizada una selección de los artículos a partir de criterios de inclusión y exclusión de la pesquisa. En seguida, a partir de la lectura de eses artículos seleccionados fueron identificados instrumentos de evaluación utilizados en la metodología de las pesquisas. Como resultado fueron encontradas 21 referencias de instrumentos de evaluación con origen extranjera y nueve referencias de instrumentos de evaluación con origen nacional, de los cuales un breve perfil fue trazado. La metodología utilizada en esta pesquisa contribuyó para identificar los instrumentos de evaluación existentes y disponibles para uso del pedagogo y para evidenciar cómo la revisión sistemática puede ser utilizada en ese tipo de pesquisa.

Palabras-clave: Educación. Instrumentos de evaluación. Revisión sistemática.

\section{Introdução}

É certo que o uso de instrumentos de avaliação pode auxiliar o professor a perceber as características de seus alunos (SILVA; MANZINI, 2011). Sabe-se, também, que há instrumentos de avaliação utilizados na área da Educação que têm o seu uso restrito ao profissional com formação na área da saúde. Diante disso, o objetivo do presente estudo foi identificar instrumentos de avaliação com enfoque educacional que permitam o seu uso por profissionais formados em pedagogia.

Uma revisão sistematizada foi realizada para identificar publicações nacionais e internacionais de instrumentos de avaliação com enfoque educacional. A revisão sistematizada foi definida por Sampaio e Mancini (2007) como uma pesquisa que utiliza a literatura como fonte de dados sobre um tema específico.

É importante salientar que a revisão sistematizada é um procedimento utilizado que pode atender ao objetivo da revisão bibliográfica, mas revisão sistemática e revisão bibliográfica não são sinônimas.

Na pesquisa bibliográfica a principal característica é a busca, seleção e fichamento de documentos relacionados a um número extenso de fontes (livros, artigos, teses, etc.). Em outras palavras, é como se fosse realizada uma varredura na literatura publicada sobre o assunto desejado com a finalidade identificar o maior número de publicação sobre um determinado assunto (MACEDO, 1994; MARCONI; LAKATOS, 2001). Nessa revisão, não se identifica um objetivo de pesquisa para produzir um novo conhecimento, pois o pesquisador, no caso, deverá tentar apreender o que há de publicação sobre o assunto em pauta. Na revisão sistemática, a principal característica não é a extensão e diversidade de fontes de coleta de dados, mas o rigor em que a busca é planejada e realizada, a fim de disponibilizar o material que atenda ao objetivo da pesquisa.

Salvador (1973, p. 47), em sua obra sobre métodos e técnicas de revisão bibliográfica, já dizia que realizar essa pesquisa (bibliográfica) “[...] é uma tarefa estafante, quase braçal, que exige muita paciência e persistência, como também certos conhecimentos e certas técnicas”. Apesar de Salvador ter se referido à revisão bibliográfica e, com certeza, pela data de publicação não ter considerado como fonte principal a busca virtual, essa passagem remetida ao contexto atual ainda é verídica. A relativa facilidade em ter acesso a inúmeras pesquisas em pouco tempo de busca virtual e o acesso rápido às bases de busca das bibliotecas exigem que o conjunto de procedimentos na busca de dados seja rigorosamente planejado, seja na pesquisa bibliográfica, 
seja na revisão sistemática. Caso contrário, o pesquisador corre o risco de não conseguir selecionar as pesquisas relevantes para o seu trabalho entre tantas disponíveis e perder-se no processo metodológico.

Esse planejamento na revisão sistemática foi chamado por Galvão, Sawada e Trevizan (2004) de protocolo. O pesquisador deve prever com rigor no protocolo a delimitação do tema, critérios de inclusão e exclusão de pesquisas (para refinar a busca frente à grande quantidade de pesquisa disponível, principalmente no sistema virtual), critérios de armazenamento e salvamento das pesquisas, leitura e fichamento de cada pesquisa. No ambiente virtual, há que se considerar ainda em qual base de dados a busca será realizada e quais palavras-chave disponibilizarão pesquisas que atendam ao objetivo do estudo a ser realizado.

O rigor do protocolo pode ser observado nos procedimentos de pesquisas que utilizaram a revisão sistematizada. Em pesquisas cujo tema tem um número menor de publicação, é possível utilizar mais de uma fonte de dados. Padilha et al. (2011) realizaram uma revisão sistemática para identificar pesquisas relacionadas a jovens mães e abandono escolar. Apesar de não estar escrito claramente no texto, a experiência dos pesquisadores com o tema e com o tipo de pesquisa é evidenciada pelos procedimentos realizados. Para realizar a busca, os pesquisadores definiram cinco bases de dados. Em quatro, eles utilizaram duas palavras-chave em português, excluindo uma terceira (gravidez na adolescência and evasão escolar and not educação sexual) e em uma base de dados eles utilizaram as mesmas palavras em inglês. A seleção do material considerou pesquisas publicadas em português, espanhol e inglês. Como resultado, os autores encontraram 19 artigos, dos quais quatro foram desconsiderados por não ter relação com o tema pesquisado. A análise foi qualitativa e o resultado foi descrito sobre cada estudo dividido por país de origem e ano de publicação. Observa-se na pesquisa de Padilha et al. (2011) que os critérios de inclusão e exclusão metodológica foram adequados para selecionar, entre o resultado da busca, os artigos que atendiam ao objetivo do estudo.

Em estudos cujo tema é amplamente pesquisado, os critérios de inclusão e exclusão devem ser bem definidos para possibilitar a filtragem das pesquisas que interessam ao pesquisador. Outra maneira de limitar a busca por um tema, seja pelo grande número de publicação, seja por considerar um período mais relevante ao tema pesquisado, é considerar um período de tempo específico para realizar a pesquisa. A delimitação do período deve estar enraizada à relevância dos fatos históricos ocorridos em relação ao tema, evitando que essa escolha seja feita sem critério, apenas para diminuir a quantidade de material publicado no período.

Em relação à análise dos dados, desde que responda ao problema de pesquisa, além da análise qualitativa, os resultados podem ser descritos a partir da sua quantificação e construção de gráficos. Silva, Braccialli e Manzini (2009) realizaram uma pesquisa com o objetivo de identificar instrumentos de publicação internacional com objetivo de avaliar a capacidade física e motora de membros superiores em criança e/ou adolescente ( 0 a 16 anos de idade) com paralisia cerebral. Para isso, os autores selecionaram uma base dados e realizaram a pesquisa a partir de uma palavra-chave que resultou em 323 referências. Para identificar as pesquisas pertinentes ao tema pesquisado, os autores elencaram critérios de inclusão e exclusão que resultou em 32 pesquisas. A maneira com que os resultados foram analisados permitiu que os dados sobre: 1) ano de publicação, 2) nome do periódico, 3) instrumentos de avaliação utilizados nos artigos e 4) número de vezes em que cada 
instrumento foi utilizado nas pesquisas fossem demonstrados em forma de gráficos. É importante salientar na pesquisa de Silva, Braccialli e Manzini (2009) que a revisão sistematizada foi utilizada para identificar quais instrumentos de avaliação de membro superior que foram utilizados nas pesquisas e não apenas identificar as pesquisas que utilizaram determinado instrumento de avaliação. Dessa maneira, além de selecionar as pesquisas, os autores tiveram ainda que identificar os nomes dos instrumentos de avaliação mencionados na metodologia de cada artigo e, a partir deles, realizar uma busca não sistemática para traçar um perfil de cada instrumento.

Diante da eficácia em utilizar a revisão sistematizada para identificar pesquisas sobre um tema específico, o atual estudo realizou uma revisão sistematizada que identificou referências de instrumentos de avaliação com enfoque educacional com acesso irrestrito ao professor.

\section{Método}

A metodologia deste trabalho seguiu os procedimentos para realizar uma revisão sistemática com análise qualitativa. Para atender aos objetivos de busca foram selecionadas duas bases de dados: 1) portal da pesquisa e 2) scielo. A busca iniciou-se na data de abril de 2010 e terminou em agosto de 2010. A metodologia detalhada, a análise e os resultados estão descritos a seguir.

\section{Instrumentos de publicação internacional: metodologia e análise dos dados}

Para identificar os instrumentos de avaliação com origem estrangeira, inicialmente, foi definida uma palavra-chave para selecionar a busca pelos periódicos online na base de dados portal da pesquisa. A palavra escolhida foi test, a escolha se justificou pelos dados de um estudo feito por Silva, Braccialli e Manzini (2009). Aquele estudo demonstrou que as palavras measure (avaliação) e assessment (avaliação) referiam-se a avaliação de provas acadêmicas com objetivos de questioná-las em relação a sua eficácia no sistema educacional e não para uso de instrumento com o objetivo de avaliar as características (acadêmicas, sociais, motoras) do aluno. Além da palavra test optou-se por acrescentar a palavra children. O intuito foi refinar a pesquisa, já que o objetivo pela revisão da literatura era recuperar instrumentos de avaliação de alunos com idade entre seis e dez anos.

As palavras-chave recuperaram o total de seis periódicos com artigos publicados em: Academic OneFile (494 artigos); Francis (20 artigos); Journals@Ovid Full-Text (990 artigos); Wilson Education Full Text (4447 artigos); Wilson Humanities Full Text (116 artigos) e Wilson Social Sciences Full Text (4013 artigos), sendo necessário identificar quais entre esses poderiam auxiliar na atual pesquisa.

Já de início, foram excluídos os periódicos Academic OneFile e Francis por não permitirem o acesso completo aos periódicos, apenas à bibliografia dos artigos. Em seguida foram excluídos: o Journals@Ovid Full-Text, pois ele fazia referência à área da saúde, especificamente relacionada à medicina, não sendo objetivo da revisão bibliográfica; o Wilson Humaniteis por possuir artigos da área da arte, música, literatura, línguas, história, filosofia, folclore e o Wilson Social Sciences, por conter artigos das ciências sociais, sociologia, psicologia, ciências políticas, administração, crimes e antropologia. O periódico que mais abrangeu o objetivo da pesquisa foi a Wilson Education por conter artigos relacionados à Educação. 
Para atender ao objetivo da pesquisa, optou-se por pesquisar no periódico os subitens Tests and scales / Validity / Construct validity. Para ter acesso aos artigos, optou-se por selecionar a opção text full pdf. Com esse comando, o periódico disponibilizou os artigos que disponibilizavam o acesso completo ao texto, excluindo os que disponibilizavam apenas as referências e os com acesso ao texto completo de maneira restrita. O resultado da busca foi um total de 309 artigos.

Para identificar entre os 309 artigos os que utilizaram em sua metodologia instrumento de avaliação pedagógica e, consequentemente, disponibilizaram a referência do instrumento, foi necessário fazer uma leitura flutuante baseada em Bardin (1977). Inicialmente definiu-se com critério de inclusão: artigos que fizessem menção à avaliação de crianças e/ou alunos. E como critérios de exclusão: 1) artigos que fizessem menção à avaliação de adultos; 2) artigos que fizessem menção à avaliação de alunos do ensino superior e 3) artigos que não atendessem ao critério de inclusão.

Atendendo aos critérios de inclusão e de exclusão, restaram 85 artigos que foram analisados, e um total de 115 instrumentos de avaliação pedagógica foram identificados nas metodologias dos artigos. É importante salientar que os instrumentos que não admitiam a sua administração por professor foram excluídos do resultado da atual pesquisa. Para exemplificar esse critério, consideremos o instrumento Diferencial Habilite ScalesSecond Edition (DAS- II) de autoria de Elliott (2007a). O DAS foi desenvolvido nos Estados Unidos com o objetivo de avaliar as habilidades cognitivas de crianças a partir de dois anos e seis meses, até adolescentes de 17 anos e onze meses, considerando a criança em idade escolar a partir de cinco anos completos. Como critério de utilização desse instrumento é necessário que o profissional seja formado em psicologia. Assim, apesar de o instrumento avaliar, entre outras coisas, habilidades necessárias para o seu desenvolvimento escolar, ele foi excluído.

A partir dos nomes dos instrumentos, foi necessário realizar uma nova pesquisa não sistematizada para identificar características específicas de cada instrumento: a) o seu objetivo; b) população avaliada; c) avaliador e d) dados sobre a avaliação propriamente dita.

Essa nova busca permitiu que um perfil de cada instrumento fosse traçado e descrito como resultado dessa pesquisa.

\section{Instrumentos de publicação internacional: resultados}

Na literatura estrangeira há um vasto número de publicações de instrumentos de avaliação elaborados para avaliar várias características do aluno e do ambiente escolar ${ }^{1}$.

Na área da linguagem e comunicação foi encontrado o Structured Photographic Expressive Language Test (SPELT-3), que é um instrumento para avaliar crianças com problemas de linguagem na faixa etária de quatro anos e zero mês até nove anos e onze meses. Essa avaliação tem o objetivo avaliar problemas nas estruturas sintáticas e morfológicas da linguagem da criança. O seu formato inclui um livro com 54 imagens fotográficas coloridas com cenas do cotidiano para estimular a criança na realização da avaliação, um manual para guiar a aplicação do instrumento e um formulário para analisar o teste. A primeira edição foi publicada no ano de 1983 e, a partir dessa data, foram reelaboradas edições

\footnotetext{
${ }^{1}$ É importante destacar que a utilização de qualquer instrumento depende da sua validação em relação à população específica, por tanto, a utilização de qualquer instrumento estrangeiro depende da existência de uma tradução e adaptação feita para atender as características da população brasileira.
} 
no ano de 1991 e 1995. O processo não exige uma formação específica ao avaliador e o tempo estimado para a sua administração é de 15 a 25 minutos. Em relação ao ambiente de aplicação, é necessário um local quieto e sem muitos estímulos visuais. A estratégia para a avaliação que deve ser seguida pelo aplicador é a de oferecer estímulos orais auxiliando a demonstração do livro com as fotografias, como, por exemplo, a pergunta “ o que há na foto que você está vendo?”, entre outras (HAYWARD et al., 2008).

$\mathrm{Na}$ área do comportamento, foram encontrados seis instrumentos para avaliação do aluno em diversos contextos.

O Social Skills Rating System (SSRS-T) (GRESHAM; ELLIOTT, 1990) Is training available? é um instrumento com o objetivo de avaliar crianças e adolescentes entre a faixa etária de três a dezoito anos com problemas de comportamento e habilidades interpessoais. $\mathrm{O}$ tempo médio previsto para a avalição é de 10 a 25 minutos e qualquer pessoa que conheça o avaliado e saiba preencher o instrumento pode fazer a avaliação.

O Teacher's Report Form (TRF) (ACHENBACH, 1991) é um instrumento no qual o professor avalia a agressividade e os problemas de conduta de alunos entre a faixa etária de seis a dezoito anos. O tempo médio previsto para a sua aplicação é de 15 a 20 minutos. $\mathrm{O}$ instrumento pode ser utilizado pelo professor com o título de mestre.

A Escala Motivation Assessment Scale (MAS) é um instrumento que avalia e identifica os estímulos motivacionais que geram e/ou mantêm os problemas de comportamento no indivíduo (DURAND; CRIMMINS, 1992). Esses estímulos foram divididos em cinco categorias: 1) social; 2) atenção; 3) tangíveis; 4) fuga e 5) estímulos sensoriais. O instrumento é composto por 16 exemplos de situações em que o comportamento pode ocorrer.
O avaliador irá ler cada exemplo, avaliar e indicar na folha de avaliação a frequência em que o comportamento ocorre, podendo variar de nunca até sempre. $\mathrm{O}$ resultado da avaliação deve ser analisado e contabilizado, e os dados diagnosticam as situações reforçadoras dos problemas de comportamento. Não há restrinção quanto à formação do avaliador, apenas que seja uma pessoa que conheça o aluno e não tenha dificuldade para preencher a ficha de avaliação, pois, será preciso identificar as situações em que o problema de comportamento estão ocorrendo.

O Penn Interactive Peer Play Scale (Pipps) é um instrumento de avaliação comportamental que possui duas escalas. A escala para avaliação feita pelos professores, que deve ser administrada na escola infantil (FANTUZZO et al., 1996) e a escala para ser avaliada pelos pais, que deve ser administrada em casa (FANTUZZO; MENDEZ; TIGHE, 1998). O objetivo das duas escalas é avaliar crianças que vivem em áreas urbanas de alto risco. Cada escala possui 32 itens avaliativos com uma escala do tipo Likert, que deve ser preenchida de acordo com a observação do comportamento da criança durante o jogo livre.

O Sutter-Eyberg Student Behavior Inventory-Revised (SESBI-R) é um instrumento que foi elaborado para avaliar problemas de comportamento em crianças e jovens entre a faixa etária de dois a dezesseis anos (EYBERG; PINCUS, 1999). Eyberg Inventário do Comportamento Infantil \& Eyberg Student-Sutter O tempo médio para a avaliação está previsto para cinco minutos e pode ser feita pelo professor que responde ao questionário.

A Scale for Assessing Emotional Disturbance (SAED) (EPSTEIN; CULLINAN, 1998) foi desenvolvida para avaliar e identificar problemas emocionais e/ 
ou dificuldade de comportamento no contexto educacional de crianças e adolescentes na faixa etária de cinco a dezoito anos. A escala abrange os seguintes domínios: incapacidade para aprender, problemas de relacionamento, comportamento inadequado, tristeza ou depressão, sintomas físicos ou medos, problemas sociais e outras competências. A avaliação pode ser feita por qualquer pessoa que conheça o aluno e saiba preencher a escala, e o tempo médio previsto para a sua aplicação é de 30 a 50 minutos.

A The Play Observation Scale (RUBIN, 2001) conhecida pelas siglas POS é uma escala que pode ser utilizada com o propósito de identificar crianças extremamente tímidas e/ou agressivas, para estudar as associações comportamentais, tais quais, as relações de apego, parentalidade e as interações das crianças com seus pares, incluindo crianças com deficiência. A avaliação deve ser realizada no ambiente escolar, e os domínios avaliados pela escala são: 1) jogo social, com as subcategorias de jogo solitário, jogo paralelo, jogo em grupo; 2) jogo cognitivo, com as subcategorias de jogo social, jogo construtivo, exploração, jogo de faz de conta, jogos com regras, comportamento ocioso, comportamento de observação do outro, transição de uma atividade para outra, interação por meio da conversação, agressão, brincadeira de queda, observação da brincadeira dos outros, jogos com ansiedade e jogos fora da sala. O avaliador deve gravar o comportamento da criança para uma posterior análise, sendo que o manual sugere que um período de cinco minutos por dia é o suficiente, e, para completar a avaliação, é sugerida uma observação de no mínimo de 15 minutos. As interações serão avaliadas pelos códigos positivo (+), neutro (0) ou negativo (-) na própria escala. Não há restrinção no uso da escala, e qualquer profissional com experiência na área pode utilizá-la.
Discussion Há também instrumentos para avaliar a área cognitiva para crianças da área da educação especial.

\section{A escala Childhood Autism Rating} Scale (CARS), que foi traduzida e validada para a cultura brasileira no ano de 2007 por Alessandra Marques Pereira (PEREIRA, 2007), é um instrumento para avaliar e identificar a criança autista da criança não autista com deficiência intelectual (SHOPLER et al., 1988). Nas crianças autistas, o escore permite identificar o autismo leve do autismo severo, e o seu uso é indicado para criança a partir dos dois anos de idade. $\mathrm{O}$ avaliador, que pode ser um profissional da área da saúde ou um profissional da área da educação, avalia 15 domínios da criança e indica a pontuação de acordo com o escore mais condizente com a sua avaliação, que pode variar de um (dentro dos limites da normalidade) até quatro (sintomas autistas graves). Os domínios avaliados pela escala são: relações pessoais, imitação, resposta emocional, uso corporal, uso de objetos, resposta à mudança, visual, auditiva, paladar, olfato e tato, medo ou nervosismo, comunicação verbal, comunicação não-verbal, nível de atividade, nível e consistência da resposta intelectual e impressões gerais. A análise dos dados segue o seguinte critério: de 15 a 30 pontos (sem autismo), de 30 a 36 pontos (autismo leve - moderado) e de 36 a 60 pontos (autismo grave). O instrumento não possui kit com materiais concretos, a avaliação deve ser feita de modo que o avaliador faça a observação da criança e quantifique seus comportamentos de acordo com os domínios da escala.

Para avaliar a área ortográfica, identificaram-se três instrumentos: 1) Developmental Spelling Analysis; 2) Astronaut Invented Spelling Test e o 3) Handwriting Assesment Protocol. 


\section{O Developmental Spelling Analysis} (DAS) é um instrumento de avaliação que foi desenvolvido para avaliar o domínio ortográfico (GANSKE, 1999). É um material que permite que o professor identifique as dificuldades ortográficas dos alunos. O instrumento pode ser utilizado por professores experientes que interpretam os dados da avaliação como elementos que guiam o planejamento da atividade, auxiliando na dificuldade do aluno. $\mathrm{O}$ material avaliativo contém um guia avaliativo para orientar a avaliação.

O Astronaut Invented Spelling Test (AIST) é um instrumento para avaliação da consciência fonêmica e habilidades ortográficas em crianças na fase da alfabetização (NEILSON, 2003). O tempo previsto para a administração é de 10 a 15 minutos e pode ser administrado por psicólogos, fonoaudiólogos e professores.

O Handwriting Assesment Protocol (POLLOCK et al., 2008) tem o objetivo de identificar dificuldades na caligrafia de crianças em nível primário. A primeira versão foi elaborada em 1994, em seguida houve (re) elaboração nos anos de 2006 e 2006 até a publicação de 2007. Os procedimentos para avaliação são: 1) registro prévio (conversa com a família e com o professor da criança); 2) observação do aluno em sala de aula; 3) observação complementar (sobre as características físicas e emocionais do aluno); 4) atividades para avaliação da escrita que envolve as áreas: a) memória; b) leitura (com estímulo longe e perto do aluno) e escrita; c) ditado; d) escrita de sentenças sobre tema escolhido pelo aluno. A avaliação deve ser feita, preferencialmente, no ambiente escolar, no entanto, há a possibilidade de ser feita no domicílio, desde que respeitadas as peculiaridades do instrumento.

Para avaliar a área do processamento auditivo, há o Lindamood Auditory Conceptualization Test (LINDAMOOD;
LINDAMOOD, 2004), um teste que avalia a capacidade do indivíduo de perceber e conceituar os sons da fala através de um meio visual. Os resultados dessa avaliação podem auxiliar fonoaudiólogos, educadores da área da educação especial e especialistas para identificar dificuldades que o aluno apresenta em relação à leitura. O kit do instrumento contém um CD de áudio que apresenta a pronúncia correta das palavras e como deve ser aplicada a avaliação. Além disso, o instrumento dispõe de um manual, livros do examinador, blocos de papel e feltros coloridos e uma caixa para o armazenamento dos materiais. O tempo médio de avaliação é de 20 a 30 minutos, e a idade para as crianças serem avaliadas é de cinco anos completos.

Para avaliar o nível de satisfação em relação à sala de aula, há o My Class Activities (MCA), que foi desenvolvido por Gentry e Gable em 2001 com o objetivo de avaliar sob a ótica dos alunos as áreas ligadas à motivação e aprendizagem: interesse, desafio, escolha e prazer em relação à sua sala de aula. Os resultados da avaliação podem auxiliar os professores a compreender como os estudantes veem suas aulas e, assim, planejar uma aula mais estimulante para todos. Nessa avaliação, os próprios estudantes, que podem ser do Ensino Fundamental e do Ensino Médio, respondem a cada um dos 31 itens da escala, os quais avaliam a sua aula com a indicação de uma resposta entre cinco pontos. Além disso, a escala tem sido pesquisada para ser utilizada para o enriquecimento curricular de alunos superdotados (PEREIRA; PETERS; GENTRY, 2010).

Para avaliar a participação de crianças e adolescentes em jogo, foi identificado o Pediatric Interest Profiles desenvolvido por Henry (HENRY, 2000), que se divide em três escalas: 1) avaliação do perfil de crianças em atividades de jogos; 2) avaliação do perfil de adolescentes em atividades de jogos; 3) 
avaliação de jovens em atividades de jogos. A escala para avaliação do perfil de crianças é ilustrada desde a categoria do jogo até a resposta avaliativa. A sua estrutura inicia-se com o nome do jogo e a ilustração, e as questões: 1) você faz essa atividade? 2) quanto você gosta da atividade; 3) com quem você faz essa atividade? A avaliação de adolescente é ilustrada apenas para demonstrar o jogo questionado. A sua estrutura inicia-se com o nome do jogo e a ilustração, e as questões: 1) você pratica essa atividade? 2) com que frequência você prática essa atividade? 3) quanto você gosta dessa atividade? 4) você acha que essa atividade é boa? 5) com quem você pratica essa atividade? A avaliação de jovem não é ilustrada. A sua estrutura inicia-se com o nome do jogo e as questões: 1) você tem interesse nessa atividade? 2) com que frequência você faz essa atividade? 3) você faz bem essa atividade? 4) quanto você gosta dessa atividade? 5) com quem você faz essa atividade? As escalas são de fácil manuseio, o próprio indivíduo pode preencher a escala com auxílio do profissional, principalmente para a análise dos dados. Segundo a autora, quanto ao perfil profissional não há restrição em relação à sua formação.

Há ainda instrumentos que avaliam o desenvolvimento infantil, conhecimentos gerais e/ou específicos.

A terceira edição da escala de desenvolvimento infantil, conhecida como Bayley-III (BAYLEY, 2005), foi elaborada para avaliar o que a autora intitulou de domínios: cognitivo, linguagem, motor, social e emocional, e comportamento adaptativo de crianças de um a quarenta e dois meses de idade. O instrumento possui um manual que inclui a parte técnica de tabulação de dados e a parte de orientação para a avaliação com o uso do instrumento; um livro com atividades e vinte e cinco formulários para registro e um conjunto de materiais concretos que formam um kit para que as crianças realizem a avaliação. A avaliação é realizada pela observação direta da criança na realização das atividades de avaliação propostas pelo instrumento. $\mathrm{O}$ ambiente escolhido para a realização da avaliação deve ser calmo e com o mínimo de estímulo visual possível e o tempo previsto é de quinze a vinte minutos. Em relação à formação do profissional, a administração do instrumento pode ser feita por qualquer profissional, desde que possua experiência em avaliação individual de alunos.

O Peabody Individual Achievement Test-Revised (PIAT-R;) foi desenvolvido por Dunn e Markwardt em 1970. Os alunos devem ser avaliados individualmente, respeitando a faixa etária de cinco anos a vinte dois anos e onze meses, incluindo crianças com deficiências graves, e o tempo médio de administração está previsto para uma hora. O objetivo da avaliação é relacionado ao desempenho individual acadêmico e abrangem as áreas de conhecimentos gerais do aluno (100 itens avaliativos), a área da leitura (182 itens avaliativos), ortografia (100 itens avaliativos) e matemática (100 itens avaliativos).

\section{O Early Screening Inventory-} Revised (ESI-R) é um instrumento que foi desenvolvido para identificar crianças de três a seis anos que podem precisar de serviços de educação especial para auxiliá-las na área acadêmica (MEISELS, 1997). O teste abrange avaliação nas áreas motora e visual, desenvolvimento adaptativo, linguagem e cognição. O tempo previsto para a administração do instrumento está previsto para quinze a vinte minutos e os avaliadores podem ser professores, psicólogo ou profissional da área da saúde, desde que tenham o treinamento para a utilização do instrumento.

OO Wide Range Achievement Test, 3rd ed. Wide Range Achievement Test or WRAT-3 is a screening test that can be administered to determine if a more comprehensive 
achievement test is needed. (WRAT-3) é um instrumento que avalia competências da área da leitura, aritmética e ortografia (JASTAK; WILKENSON, 1993). The test covers ages from five to 75 years old and takes approximately 30 minutes to administer. O teste cobre a faixa etária dos cinco aos setenta e cinco anos e leva aproximadamente 30 minutos para ser administrado. A avaliação ocorre com a prova de leitura, que tem que ser avaliada de maneira individual, e com a avaliação da aritmética e da ortografia, que podem ser realizadas em grupo.

O Assessment of Basic Language \& Learning Skills é um instrumento para a avaliação das capacidades básicas da linguagem, acadêmicas, de autoajuda e habilidades motoras de crianças na faixa etária de três a nove anos de idade. Segundo o autor Partington (2006), o objetivo da avaliação é que seus resultados sejam utilizados para direcionar o planejamento de um currículo individualizado para atender as necessidades do aluno. A avaliação é realizada por observação do comportamento da criança em cada área de habilidade. $\mathrm{O}$ avaliador fornecerá estímulos que podem ser verbal e/ou física, e é o nível de ajuda e o comportamento da criança que determina o resultado da avaliação.

O School Observation Code (ADHD) é um instrumento que foi desenvolvido para avaliar os sintomas do transtorno de déficit de atenção com hiperatividade (TDH) (GADOW; NOLAN, 1996). A avaliação é feita por observação da criança no contexto escolar, incluindo sala de aula, contexto de recreação, lanchonete e parque. O tempo previsto para a sua aplicação é de 30 minutos, e a faixa etária da criança não foi informada, apenas foi indicado que o aluno deve estar matriculado da pré-escola ao sexto ano do Ensino Fundamental.
Instrumentos de publicação nacional: metodologia e análise dos dados

Para realizar o levantamento bibliográfico nacional optou por fazer uma busca na base de dados Scielo com a palavra-chave desempenho, já que a palavra avaliação recuperava apenas artigos sobre prova acadêmica, o que não abrangia o objetivo da atual pesquisa.

A base de dados disponibilizou outras palavras-chave relacionadas à palavra desempenho: 1) desempenho acadêmico (com 14 artigos); 2) desempenho cognitivo (três artigos); 3) desempenho do aluno (um artigo); 4) desempenho escolar (27 artigos); 5) desempenho escolar matemática (um artigo) e 6) desempenho psicomotor (oito artigos), totalizando 54 artigos de origem nacional.

Para identificar entre os 54 artigos os que possuíam referência de instrumento de avaliação pedagógica, seguiu-se a metodologia utilizada anteriormente na busca por instrumentos de origem internacional.

Os 54 artigos foram analisados com o objetivo de selecionar as pesquisas que atendessem os critérios de inclusão: artigos que fizessem menção à avaliação de crianças e/ou alunos. E os critérios de exclusão: 1) artigos que fizessem menção à avaliação de adultos; 2) artigos que fizessem menção à avaliação de alunos do ensino superior; 3) artigos que não atendessem ao critério de inclusão, resultando em 11 pesquisas com referências de instrumentos de avaliação.

A análise dos 11 artigos identificou um total de nove instrumentos de avaliação, que foram descritos conforme o seu objetivo de uso e suas principais características.

Semelhante ao procedimento na busca por instrumentos internacionais, a partir dos nomes dos instrumentos, foi realizada uma nova pesquisa não sistematizada para identificar características específicas de cada 
instrumento: a) o seu objetivo; b) população avaliada; c) avaliador; d) dados sobre a avaliação propriamente dita.

Um perfil de cada instrumento foi traçado e descrito como resultado dessa pesquisa.

\section{Instrumentos de publicação nacional: resultados}

Na literatura nacional foram encontrados instrumentos para avaliar: desempenho acadêmico em diferentes níveis escolar; o nível de operatoriedade da criança a partir dos três anos de idade; o autoconceito em crianças e jovens, entre outros.

O Teste de Desempenho Escolar (TDE) (STEIN, 1994) foi desenvolvido para avaliar crianças do $2^{\circ}$ ao $7^{\circ}$ ano do Ensino Fundamental nas habilidades da área da escrita, aritmética e leitura. O instrumento contém um manual para aplicação e interpretação, cinco cadernos para a avaliação, uma ficha para cada um dos subtestes de leitura, aritmética e escrita. Qualquer profissional que atue na área da educação pode utilizar o instrumento.

A Coleção Papel de Carta (CHAMAT, 1997) foi elaborada para avaliar as dificuldades de aprendizagem das crianças com idade entre seis a onze anos. A avaliação pode ser feita de maneira individual ou coletiva, com o tempo médio de aplicação de uma hora. $\mathrm{O}$ instrumento é composto por, segundo a autora, seis lâminas com ilustração de desenhos, as quais são apresentadas uma a uma para a criança contar uma história relacionada com a ilustração apresentada.

O instrumento intitulado de Os Contos de Fadas e a Psicopedagogia (LIMA, 1998) foi elaborado para avaliar crianças em fase de alfabetização. Não há limite de faixa etária, apenas a condição da criança estar em fase de alfabetização. A aplicação do instrumento deve ser individual e o tempo previsto é de cinco a dez minutos.

O Método Horizontes (MARTINS, 2003) foi elaborado com o objetivo de avaliar e identificar crianças da faixa etária de cinco a oito anos que estão preparadas para serem alfabetizada. As áreas de avaliação abrangem: a área motora, o nível de percepção e discriminação, nível de independência, nível de linguagem, nível de reconhecimento das cores, conhecimento das formas e capacidade de relacionar quantidades.

A Escala de Avaliação do Comportamento Infantil para o Professor (BRITO, 2006a) tem o objetivo de avaliar áreas acadêmicas relacionadas ao desempenho na aritmética, ditado e leitura, além de avaliar características da família e dificuldades acadêmicas em geral. Para fazer a avaliação, o professor observa o comportamento da criança e/ ou adolescente (entre a faixa etária de quatro a catorze anos) e avalia seus comportamentos de acordo com o que é proposto na escala. A avaliação deve ser feita de modo individual, e não há limite ou previsão de tempo para que a avaliação seja concluída.

Na área das funções operatórias, há a Coleção Aprendizagem e Nível De Operatoriedade: Três Porquinhos (CHAMAT, 2006). Essa avaliação foi elaborada com o objetivo de avaliar o nível de operatoriedade da criança a partir dos três anos de idade. $\mathrm{O}$ tempo médio para a sua aplicação é de uma hora, mas não há limites para a sua aplicação. O instrumento contém oito pranchas relacionadas à história dos três porquinhos. Com a orientação do avaliador, a criança deve contar uma história para cada prancha apresentada, e a análise dos resultados é feita de uma maneira qualitativa.

A Escala de Auto-Conceito InfantoJuvenil (SISTO, 2004) tem o objetivo de avaliar os níveis de autoconceito de crianças 
e jovens na faixa etária entre oito a dezesseis anos. O tempo médio de avaliação está previsto entre cinco e dez minutos. O instrumento abrange as áreas de interação entre a pessoa avaliada e o seu ambiente, dividido em autoconceito pessoal, autoconceito escolar, autoconceito familiar e autoconceito social.

Na área da leitura, Provas de Avaliação dos Processos de Leitura (PROLEC) (CAPELLINI; OLIVEIRA; CUETOS, 2010) é um instrumento para avaliação de crianças disléxicas e com dificuldade de aprendizagem. O instrumento contém dez provas que abrangem áreas do desenvolvimento da leitura na criança. Os profissionais da área da pedagogia, fonodiologia e psicologia estão aptos para aplicar o instrumento.

O Teste do Span de Cores (BRITO, 2006b) foi elaborado com o objetivo de avaliar crianças e adolescentes, na faixa etária de 8 a 16 anos, abrangendo as áreas de apresentação visual e resposta visual, apresentação visual e resposta verbal, apresentação verbal e resposta visual, apresentação verbal e resposta verbal. A avaliação deve ser feita individualmente, e o tempo médio previsto para a aplicação é de 15 a 30 minutos.

\section{Considerações Finais}

A revisão sistematizada foi utilizada na metodologia deste trabalho para identificar artigos com referências de instrumentos de avaliação pedagógica de publicação nacional e estrangeira que permitissem que o seu uso fossem feito por professores.

Em relação aos resultados, observa-se que na literatura estrangeira foram encontrados 21 instrumentos de avaliação com diferentes enfoques: instrumento na área da linguagem e comunicação (1); na área cognitiva (1); na área de desenvolvimento infantil (1); para avaliar o processamento auditivo (1); o nível de satisfação em relação à sala de aula (1); a participação de crianças e adolescente em jogos e em hiperatividade (1); instrumentos para avaliar a ortografia (3); instrumentos para avaliar o desempenho acadêmico (4); instrumentos para avaliar o comportamento (7).

Na literatura brasileira foram encontrados nove instrumentos de avaliação: instrumento para avaliar o nível de operatoriedade (1); autoconceito (1); leitura (1); resposta verbal e visual (1); para avaliar a área acadêmica em diferentes níveis e focos (5).

Os resultados desta pesquisa contribuem para que professores conheçam que há instrumentos que podem ser utilizados por eles com diferentes objetivos de avaliação, em destaque para os de origem brasileira, já que os de origem estrangeira dependerão de adaptação cultural.

Não há como generalizar, mas os dados demonstraram que há uma quantidade superior de publicação de origem estrangeira que utilizaram instrumentos de avaliação (309 artigos) quando comparadas com as de origem nacional (54 artigos), considerando, especificamente, as duas fontes de dados pesquisadas.

É importante salientar que os resultados foram oriundos da escolha metodológica incluindo a escolha da base de dados e das palavras-chaves. Em outras palavras, se houver uma nova pesquisa que considere outro protocolo prevendo outras bases de dados, assim como outras palavras-chave, o resultado certamente diferirá do encontrado nesta pesquisa. Talvez essa versatilidade seja uma das características principais da revisão sistemática de dados.

\section{Referências}

ACHENBACH, T. M. Manual of the
teacher's report form and 1991 profile. 
Burlington, VT: University of Vermont, Department of Psychiatry, 1991.

BARDIN, L. Análise de conteúdo. Lisboa: Edições 70, 1977.

BEARD, K. Wide Range Achievement Test. Gale Encyclopedia of Mental Disorders, 2003. Disponível em: <http:// www.encyclopedia.com>. Acesso em: 17 jan. 2011.

BRITO, G. N. O. Escala de avaliação do comportamento infantil para o professor. São Paulo: Vetor, 2006a.

Teste do span de cores. São Paulo: Vetor, 2006b.

CAPELLINI, S. A; OLIVEIRA, A. M.; CUETOS, F. PROLEC: provas de avaliação dos processos de leitura. Itatiba - SP: Casa do Psicólogo, 2010.

CHAMAT, L. S. J. Coleção aprendizagem e nível de operacionalidade: três porquinhos. São Paulo: Vetor, 2006.

Coleção papel de carta. São Paulo: Vetor, 1997.

DUNN, L. M.; MARKWARDT, F. Peabody individual achievement test Manual. Circle Pines, Minnesota: American Guidance Service, 1970.

DURAND, V. M.; CRIMMINS, D. B. The motivation assessment scale (MAS): administration guide. Topeka, KS: Monaco and Associates, 1992.

ELLIOTT, C. D. Differential ability scales, 2nd edition: introductory and technical handbook. San Antonio, TX: The Psychological Corporation, 2008.

EPSTEIN, M. H.; CULLINAN, D. The scale for assessing emotional disturbance. Austin, TX: PRO-ED, 1998.
EYBERG, S.; PINCUS, D. Eyberg child behavior inventory \& sutter-eyberg student Behavior Inventory-Revisad: Professional Manual. Odessa, FL: Psychological Assessment Resources, 1996.

FANTUZZO, J. W. et al. The pictorial scale of perceived competence for young children: does it work with low-income urban children? Child Development, v. 67, p. 1071-1084, 1996.

FANTUZZO, J.; MENDEZ, J.; TIGHE, E. Parental assessment of peer play: development and validation of the parent version of the penn interactive peer play scale. Early Childhood Research Quarterly, v.13, p. 659-676, 1998.

GADOW, K. D.; SPRAFKIN, J.; NOLAN, E. E. ADHD school observation code. Stony Brook, NY: Checkmate Plus, 1996.

GALVÃO, C. M.; SAWADA, N. O.; TREVISAN, M. A. Revisão sistemática: recurso que proporciona a incorporação das evidencias na prática da enfermagem. Rev. Latino-am Enfermagem, v.12, n. 3, p. 549556, 2004.

GANSKE, K. The developmental spelling analysis: a measure of orthographic knowledge. Educational Assessment, v. 6, n. 1, p. 41-70, 1999.

GENTRY, M.; GABLE, R. K. My class activities: a survey instrument to assess students' perceptions of interest, challenge, choice and enjoyment in their classrooms. (Instrument). Mansfield Center, CT: Creative Learning Press, 2001.

GRESHAM, F. M.; ELLIOTT, S. N. Social skills rating system. Circle Pines, $\mathrm{MN}$ : American Guidance Service, 1990.

HAYWARD, D. V. et al. Test review: structured photographic expressive language test-3 (SPELT-3). Language, 
phonological awareness, and reading test director. Edmonton, $\mathrm{AB}$ : Canadian Centre for Research on Literacy, 2008, p 1-7. Disponível em: <http://www.uofaweb. ualberta.ca/elementaryed/ccrl.cfm. >. Acesso em: 5 out. 2010.

HENRY, A. D. Survey of play for children and adolescents: kid play profile; preteen play profile; adolescent leisure interest profile; therapy skill builders. United States of America, 2000.

JASTAK S.; WILKINSON G. The wide range achievement test: WRAT3. Wilmington, Delaware: Jastak Assessment Systems, 1993.

LIMA, T. Os contos de fadas e a psicopedagogia. São Paulo: Vetor, 1998.

LINDAMOOD, P. C.; LINDAMOOD, P. Lindamood auditory conceptualization test: Examiner's manual. Austin, TX: ProEd., 2004.

MACEDO, N. D. Iniciação à pesquisa bibliográfica. São Paulo: Loyola, 1994.

MARCONI, M. A.; LAKATOS, E. M. Metodologia do trabalho científico. São Paulo: Atlas, 2001.

MARTINS, N. L. B. Coleção métodos horizontes. São Paulo: Vetor, 2003.

MEISELS, S. J. et al. Early screening inventory - revised: examiner's manual. Ann Arbor, Michigan: Rebus, Inc, 1997.

NEILSON, R. The astronaut invented spelling test. Jamberoo: Author, 2003.

PADILHA, M. A. S. et al. Jovens mães e abandono escolar: uma revisão sistematizada. Rev. Enferm. UFPE on line, v. 5, n. 6, p. 1534-1540, 2011.

PARTINGTON, J. The assessment of basic language and learning skills (revised).
Pleasant Hill, CA: Behavior Analysts Inc.; 2006.

PEREIRA, A. M. Autismo Infantil: Tradução e validação da CARS (Childhood Autism Rating Scale) para uso no Brasil. 2007. 98 f. Dissertação (Mestrado em Ciências Médicas), Universidade Federal do Rio Grande do Sul. Porto Alegre, Rio Grande do Sul, 2007.

PEREIRA, N.; PETERS, S. J.; GENTRY, M. My class activities instrument as used in Saturday enrichment program evaluation. Journal of Advanced Academics (Magazine/Journal), June 22, 2010.

POLLOCK, N. et al. The mac master: handwriting assessment protocol: school of rehabilitation science: Mc Master University, 2009. Disponível em: <www.canchild.ca/ default.aspx?tabid=205-86k $>$. Acesso em: 20 jan. 2011.

POPPOVIC, A. M. Teste metropolitano de prontidão, forma $R$ de Hildreth $e$ Griffiths. São Paulo: Vetor, 1966.

RUBIN, K. H. The play observation scale: the center for children, relationships and culture, University of Maryland, College Park: Author, 2001.

SALVADOR, A. D. Métodos e técnicas de pesquisa bibliográfica. Porto Alegre: Sulina, 1977.

SAMPAIO, R. F.; MANCINI, M. C. Estudo de revisão sistematizada: um guia para síntese criteriosa da evidencia científica. Rev. Bras. Fisioter., São Carlos, v.11, n.1, p. 83-89, 2007.

SCHOPLER, E. et al. The childhood autism rating scale. Los Angeles: Western Psychological Services, 1988.

SILVA, M. O.; BRACIALLI, L. M. P.; MANZINI, E. J. Instrumentos de avaliação 
motora em crianças e adolescentes ( 0 a 16 anos) com paralisia cerebral. 32f. In: IV Seminário Nacional Promoção de inclusão mediada pelas tecnologias assistivas, Curitiba. Anais... Curitiba: UTP/UEPA/ UFBA, p. 27-28, 2009.

SILVA, M. O.; MANZINI, E. J. Avaliação pedagógica do aluno com deficiência e a prática do professor de Educação Especial. In: $10^{\circ}$ Encontro de pesquisa em educação da região sudeste, Rio de Janeiro. Anais... Rio de Janeiro: CNPq, Anped, 2009.

SISTO, F. F. Coleção EAC-IJ: escala de auto-conceito infanto-juvenil. São Paulo: Vetor, 2004.

STEIN, L. TDE - Teste de desempenho escolar. São Paulo: Casa do Psicólogo, 1994. 